\title{
Evaluación de una estrategia de educación ambiental sobre el conocimiento de la fauna nativa en el marco de la taxonomía folk
}

\author{
Evaluation of an Environmental Education Strategy on the Knowledge of Native Fauna \\ Within the Framework of Folk Taxonomy
}

\section{Avaliação de uma estratégia de educação ambiental sobre o conhecimento da fauna nativa no âmbito da taxonomia folk}

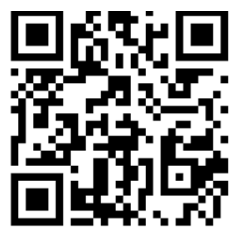

Gilbert Enrique Salas-López

Universidad de Ciencias Aplicadas y Ambientales

Bogotá, Colombia

gilbert.e.salas@gmail.com

https://orcid.org/0000-0002-3453-1104

\author{
Recibido • Received • Recebido: 28 / 01 / 2019 \\ Corregido • Revised • Revisado: 04 / 08 / 2020 \\ Aceptado • Accepted • Aprovado: 19 / $11 / 2020$
}

\begin{abstract}
Resumen: Dado que la taxonomía folk se relaciona con una clasificación innata de los organismos, esta podría ser la base para diseñar, implementar y evaluar estrategias de educación ambiental enfocadas en la biodiversidad y su conservación. El presente estudio evaluó, en el marco de la taxonomía folk, una intervención de educación ambiental basada en el juego para mejorar el conocimiento de las especies nativas de Colombia. 291 estudiantes de $4^{\circ}$ - $^{\circ}$ grado de 11 colegios públicos de Cundinamarca jugaron ad libitum durante 4-5 semanas en el tiempo del descanso escolar, sin la presencia de personal de tutoría. Se le pidió al estudiantado que enlistaran todos los animales de Colombia que conocían, pre-juego y post-juego. Los animales nativos mencionados se asignaron a las categorías taxonómicas etnobiológicas "formas de vida", "genérica" y "específica". Aunque la composición de las formas de vida no cambió, hubo una disminución en su abundancia (taxones: 2 pre-juego vs. 2 post-juego; menciones: 101 pre-juego vs. 89 post-juego) y un aumento en la composición y abundancia de las categorías genérica (taxones: 82 pre-juego y 104 post-juego; total 115; menciones: 2293 pre-juego vs. 2505 post-juego) y específica (taxones: 24 pre-juego vs. 53 post-juego; total 64; menciones: 400 pre-juego vs. 706 post-juego). Debido a la relación entre la cultura y las categorías taxonómicas etnobiológicas, la disminución en la abundancia de formas de vida, al igual que el aumento en la composición y abundancia de los taxones en las categorías genérica y específica, puede considerarse un resultado ideal para las intervenciones de educación ambiental relacionadas con el conocimiento de la biodiversidad y la identificación de especies.
\end{abstract}

Palabras claves: Educación ambiental; biodiversidad; fauna autóctona. 
http://doi.org/10.15359/ree.25-1.2

http://www.una.ac.cr/educare

educare@una.ac.cr

\begin{abstract}
Since folk taxonomy is related to the innate classification of organisms, it could be the basis for designing, implementing, and evaluating environmental education strategies focused on biodiversity and its conservation. Through folk taxonomy, this study assessed a game-based environmental education intervention designed to improve Colombian native species' knowledge. Two hundred and ninety-one (291) students from 4th to 9th grade of 11 public schools in Cundinamarca, Colombia, played ad libitum during the school recess period without a tutor. Students were asked to list all native Colombian animals they knew before playing for the first time and after having played for four to five weeks. The native animals mentioned were classified into the following ethnobiological taxonomic categories: life forms, generic, and specific. Although the composition of the "life forms" category did not change, there was a decrease in its abundance (taxa: two pre-game vs. two post-game; mentions: 101 pre-game vs. 89 post-game). Besides, there was an increase in composition and abundance in the "generic" category (taxa: 82 pre-game vs. 104 post-game; total 115; mentions: 2293 pre-game vs. 2505 post-game) and in the "specific" category (taxa: 24 pre-game vs. 53 post-game; total 64; mentions: 400 pre-game vs. 706 post-game). Because of the relation the relation between culture and the ethnobiological taxonomic categories, the decrease of abundance of taxa in the "life forms" category, and the increase of composition and abundance of taxa in the "generic" and "specific" categories, the results of this study can be considered as ideal for education interventions based on biodiversity and species identification.
\end{abstract}

Keywords: Environmental education, biodiversity, native fauna.

Resumo: Pelo fato de que a taxonomia folk está relacionada a uma classificação inata dos organismos, ela poderia ser a base para a concepção, implementação e avaliação de estratégias de educação ambiental enfocadas na biodiversidade e sua conservação. $O$ presente estudo avaliou, no âmbito da taxonomia folk, uma intervenção de educação ambiental baseada no jogo, para melhorar o conhecimento das espécies nativas da Colômbia. 291 alunos do $4^{\circ}$ ao $9^{\circ}$ ano, de 11 escolas públicas de Cundinamarca, jogaram à vontade por 4-5 semanas no recreio, sem a presença de um tutor. Os e as estudantes foram convidados a listar todos os animais da Colômbia que eles conheciam, préjogo e pós-jogo. Os animais nativos mencionados foram designados para as categorias taxonômicas etnobiológicas "formas de vida", "genérica" e "específica". Embora a composição de "formas de vida" não tenha mudado, houve uma diminuição na sua abundância (táxones: 2 pré-jogo vs. 2 pós-jogo; menções: 101 pré-jogo vs. 89 pós-jogo) e um aumento na composição e abundância das categorias "genérica" (táxones: 82 pré-jogo e 104 pós-jogo; total 115; menções: 2.293 pré-jogo vs. 2.505 pósjogo) e "específica" (táxones: 24 pré-jogo vs. 53 pós-jogo; total 64; menções: 400 pré-jogo vs. 706 pósjogo). Por causa da relação entre a cultura e as categorias taxonômicas etnobiológicos, a diminuição na abundância de "formas de vida", bem como o aumento na composição e abundância dos táxones nas categorias "genérica" e "específica", pode ser considerado um resultado ideal para as intervenções de educação ambiental relacionadas ao conhecimento da biodiversidade e identificação de espécies.

Palavras chave: Educação ambiental; biodiversidade; fauna nativa.

\title{
Introducción
}

Un papel fundamental de la educación ambiental es facilitar que la comunidad no solo comprenda los procesos biológicos y ecológicos del entorno al que pertenece, sino también que conozca y ame la biodiversidad que la rodea, de tal manera que pueda tomar decisiones 
http://doi.org/10.15359/ree.25-1.2

informadas en relación con la conservación y protección del medio ambiente. En el caso de Colombia, la educación ambiental relacionada con la biodiversidad adquiere una mayor relevancia, en particular porque el $50 \%$ de los ecosistemas del país se encuentra bajo algún grado de amenaza y existe una tendencia hacia una pérdida acelerada de la biodiversidad (Gómez y Moreno, 2016). Por consiguiente, es indispensable que se exploren y evalúen diferentes estrategias educativas que conduzcan a cambios profundos en el conocimiento y el afecto del público frente al medio ambiente en general y a la fauna nativa en particular, pues esta es un elemento que puede generar vínculos cognitivos y afectivos entre el individuo y su entorno.

Dado que la niñez es el momento ideal para implementar la educación ambiental, se diseñó un juego de cartas que utilizaba datos científicos y taxonómicos para simular las posibles relaciones tróficas entre los diferentes grupos de animales. Al evaluar este juego como estrategia de educación ambiental para mejorar el conocimiento de la fauna nativa de Colombia, se encontró que el estudiantado no solo era capaz de enlistar un mayor número de animales, sino también que se refería a ellos de una manera diferente; por ejemplo, antes de jugar era frecuente que el estudiantado mencionara simplemente "ardilla" o "rana", y después de jugar comenzaron a mencionar con mayor frecuencia "ardilla roja" o "rana dardo dorada", etc. El objetivo del presente artículo es evaluar esta mayor especificidad al referirse a los animales dentro del marco de la taxonomía folk, ya que en este marco dicho cambio tiene bases psicológicas, lingüísticas y culturales, y podría ser relevante para evaluar los efectos de las estrategias de educación ambiental sobre biodiversidad.

\section{Marco teórico}

El ser humano es, en esencia, un animal que clasifica, y su existencia ha dependido de su capacidad de reconocer y comunicar las similitudes y diferencias entre los objetos y eventos de su universo físico (Raven et al., 1971). Asimismo, parece que la capacidad de clasificar los organismos es una habilidad evolutiva, ya que cualquiera sea el trasfondo cultural, la niñez no solo reconoce y aprende los nombres de los animales que la rodean, sino también los clasifican en una taxonomía intuitiva (Anderson, 2001). En este contexto, ya que se considera que la taxonomía y la conservación van de la mano, en particular porque no puede esperarse el éxito en la conservación de organismos que no pueden identificarse (Mace, 2004), es una transición lógica abordar las estrategias educativas enfocadas en el conocimiento y conservación de la diversidad biológica, al igual que la evaluación de su efecto, en el marco de un conocimiento de la fauna inherente a la naturaleza humana, como la taxonomía folk o taxonomía tradicional. Dicha taxonomía no solo suele basarse en atributos superficiales de los seres vivos (Tun Tun, 2016), sino que corresponde a una clasificación que hace la sociedad como producto de sus necesidades (Goyenechea, 2007), y sus principios estructurales generales trascienden las diferencias entre las sociedades individuales (Berlin et al., 1973). 
http://doi.org/10.15359/ree.25-1.2

http://www.una.ac.cr/educare

educare@una.ac.cr

En cuanto al conocimiento de la biodiversidad, el público general tiene un escaso conocimiento de esta (Campos et al., 2013). De hecho, puede decirse que el problema ambiental y la pérdida de la biodiversidad relacionada tienen que ver con una crisis cognitiva, la cual es consecuencia de la "ceguera" que sufre la gente frente a los miles de seres vivos que nos rodean (Rozzi et al., 2003).

Con esta problemática de fondo, por un lado, profesionales en biología corren contra el reloj para descubrir y catalogar tantas especies como sea posible antes de que se extingan; por otro lado, en etnobiología se esfuerzan en estudiar el conocimiento y uso de las especies por parte de las diferentes culturas, antes de que estas desaparezcan. Así, el objeto de estudio para la etnobiología no es el ser vivo sino el conocimiento de este y su relación con el ser humano y, por consiguiente, las metodologías en este campo de estudio se alejan de las ciencias duras y se acercan a las ciencias blandas. De hecho, en la etnobiología, para estudiar el conocimiento de la biodiversidad y su clasificación local se utilizan métodos replicables y sistemáticos de las ciencias sociales, como las entrevistas estructuradas y, en particular, el enlistado libre (Hilgert, 2007).

En lo que se refiere a los estudios etnobiológicos, la totalidad de quienes examinaron el conocimiento y clasificación de la biota encontraron una jerarquía de términos respecto al reino animal y vegetal que constaba de, al menos, tres niveles (nivel superordinado [forma de vida], nivel básico [genérico] y nivel subordinado [específico]) (Zubin y Köpcke, 1986). Esta misma jerarquización se reconoce a nivel psicológico, ya que en la estructura de categorías mentales de los objetos concretos pueden distinguirse tres niveles: a) las categorías de los niveles más abstractos son las categorías superordinadas (por ejemplo, "mueble", "vehículo"); b) las categorías en un nivel menos abstracto con un nivel básico de abstracción (por ejemplo, "silla", "auto"); y c) las categorías por debajo del nivel básico, que son las categorías subordinadas (por ejemplo, "silla de cocina", "auto deportivo") (Rosch et al., 1976).

Así, inherentemente, el ser humano agrupa los organismos en estas categorías psicológicas/lingüísticas/etnobiológicas de manera semejante a la taxonomía científica, lo cual dio origen a la taxonomía folk. Por ejemplo, mientras la categoría genérica de la taxonomía folk (por ejemplo, laurel) es la más amplia, representa la base de esta taxonomía, es la que primero aprende la niñez y la que se observa con mayor facilidad por ser la más sobresaliente desde un punto de vista psicológico, la categoría específica (por ejemplo, laurel blanco), en caso de existir, da una idea de la importancia cultural del animal o la planta (Hilgert, 2007).

Con base en lo anterior, este estudio se centró en el análisis del cambio generado por una intervención de educación ambiental en el contexto de tres (forma de vida, genérica y específica) de las cinco (inicial, forma de vida, genérica, específica y variedad) categorías taxonómicas etnobiológicas propuestas para una taxonomía folk ideal (Figura 1) (Berlin et al., 1973). 
http://doi.org/10.15359/ree.25-1.2

Figura 1: Categorías etnobiológicas propuestas para una taxonomía folk ideal.

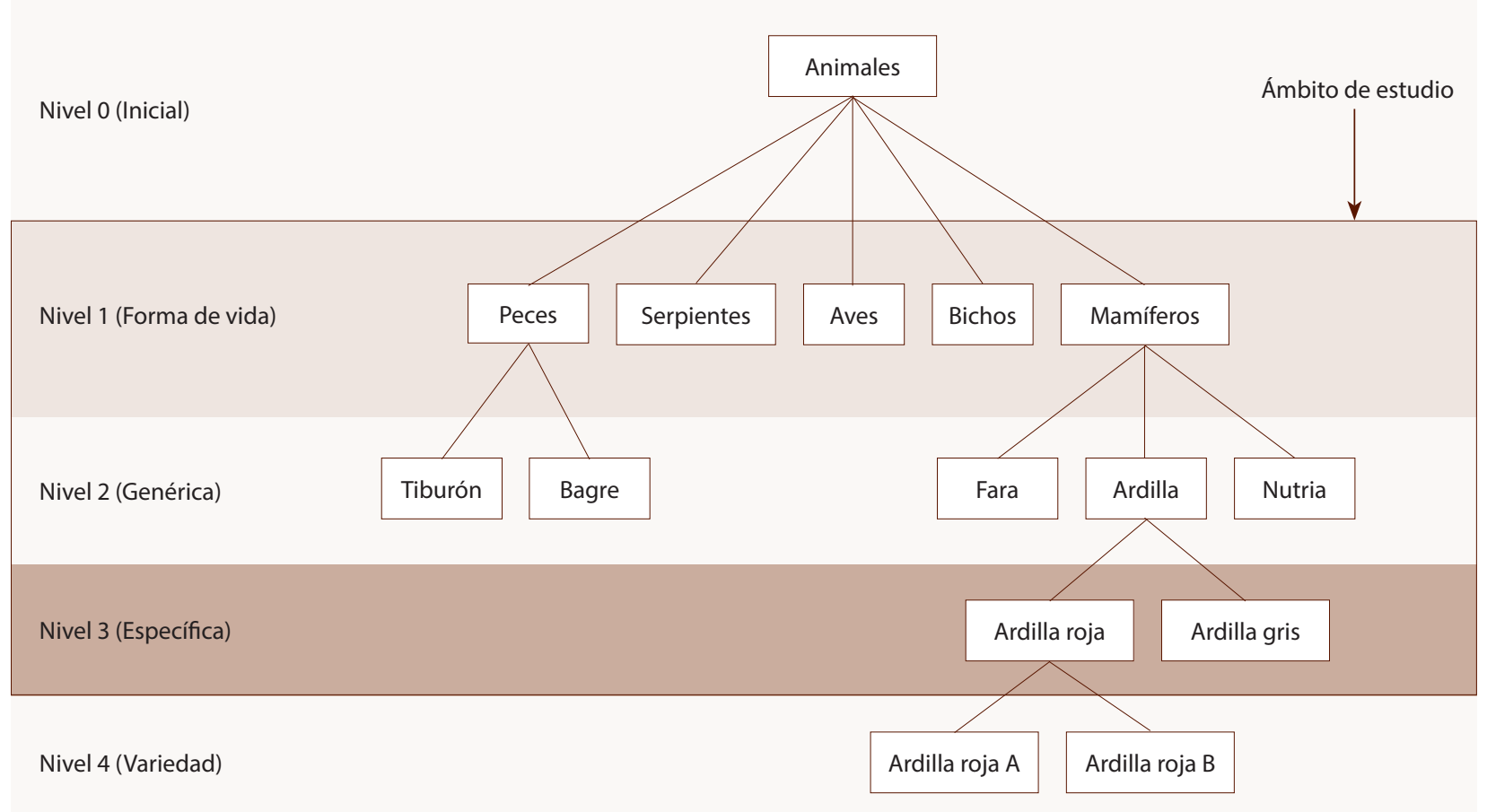

Nota: Adaptado de Berlin et al. (1973).

Con respecto al uso de juegos como herramienta de aprendizaje, el juego no solo es una parte estructural de la vida de niños y niñas, sino también una necesidad natural del ser humano (Petrovska et al., 2013). Asimismo, además de preferir un aprendizaje basado en imágenes y una información rápida, niñas y niños también se desempeñan mejor en un ambiente de recompensas, es decir, adoran los juegos (Prensky, 2006 citado en Bevilacqua et al., 2015). En este sentido, no es sorprendente que varios meta-análisis de la bibliografía publicada sobre el juego en los últimos cuarenta años hayan encontrado de modo consistente que los juegos promovían el aprendizaje y reducían el tiempo de instrucción a lo largo de diferentes edades y disciplinas (van Eck, 2006). Y en la educación ambiental, el juego se considera una herramienta idónea, ya que además de representar, de una manera simple y didáctica, el funcionamiento y la problemática de sistemas complejos, también proporciona un marco de referencia que permite tener una visión multidisciplinaria de las diferentes situaciones, y al final, el estudiantado está preparado para tomar decisiones informadas (Taylor, 1983).

\section{Materiales y métodos}

Este estudio cuasi-experimental con un diseño pre-test/post-test incluyó 291 estudiantes de $4^{\circ}$ a $9^{\circ}$ grado pertenecientes a colegios públicos rurales y urbanos de Cundinamarca (Tabla 1). 
http://doi.org/10.15359/ree.25-1.2

http://www.una.ac.cr/educare

educare@una.ac.cr

Tabla 1: Datos demográficos de la población de estudio

\begin{tabular}{|c|c|c|c|c|c|c|}
\hline \multicolumn{7}{|c|}{ Colegios rurales ( $n=143$ ) (Edad promedio 12,4) } \\
\hline Colegio & \multicolumn{2}{|c|}{ Novilleros } & Tausa & Tocancipá & Pasuncha & La Mesa \\
\hline $\mathrm{n}$ & \multicolumn{2}{|c|}{21} & 15 & 40 & 14 & 53 \\
\hline Edad promedio & \multicolumn{2}{|c|}{13,1} & 9,9 & 12,9 & 10,3 & 13,1 \\
\hline Rango de edad & \multicolumn{2}{|c|}{$11-17$} & $8-12$ & $12-14$ & $8-14$ & $11-18$ \\
\hline \multicolumn{7}{|c|}{ Colegios de Bogotá ( $n=148)$ (Edad promedio 11,8) } \\
\hline Colegio & Yomasa & Chuniza & Compartir & Tibabuyes & Marco Fidel Suárez & Villas del Progreso \\
\hline $\mathrm{n}$ & 29 & 21 & 42 & 22 & 22 & 12 \\
\hline Edad promedio & 13,9 & 11,8 & 10,5 & 11,4 & 10,6 & 14,5 \\
\hline Rango de edad & $13-17$ & $11-15$ & $8-13$ & $10-14$ & $9-13$ & $13-17$ \\
\hline
\end{tabular}

Nota: Elaboración propia.

Después de obtener los consentimientos informados de las instituciones y de los padres y madres de las personas participantes se procedió a recabar la información sobre el conocimiento de la fauna nativa de Colombia que tenía el estudiantado, para lo cual se pidió enlistar todos los animales de Colombia que conocían, tanto antes como después de 4-5 semanas de juego ad libitum durante el descanso escolar. Se escogió el enlistado libre por ser una técnica robusta y simple, donde las enumeraciones espontáneas de nombres de animales son la base para realizar estudios de los sistemas de clasificación folk (Hilgert, 2007). Asimismo, ya que este sistema de clasificación etnobiológica puede utilizarse como una herramienta para un acceso rápido a la biodiversidad (Newmaster et al., 2006, citados en Ferreira et al., 2009), se utilizó una clasificación biológica folk como marco para evaluar el conocimiento léxico del estudiantado respecto a los animales nativos (Beaudreau et al., 2011).

Los nombres de animales enunciados por el estudiantado fueron catalogados de acuerdo con la clasificación folk en las categorías "forma de vida", "genérica" y "específica". La categoría "forma de vida" estuvo constituida por las agrupaciones más grandes y más heterogéneas que se encuentran de manera regular en las taxonomías folk (peces, serpientes, aves, bichos y mamíferos) (Brown, 1979). La categoría genérica estuvo constituida por lexemas primarios (por ejemplo, nutria, garza, etc.), mientras que la categoría específica estuvo constituida por lexemas secundarios (por ejemplo, nutria gigante, garza morena, etc.) (Berlin et al., 1973; Cabral de Oliveira, 2008). En el análisis solo se incluyeron los animales pertenecientes a la fauna nativa de Colombia.

\section{Juego de cartas}

Se eligió un juego de cartas por ser un recurso conocido en la educación, cuyo uso para mejorar el aprendizaje se ha estudiado ampliamente (Turkay et al., 2012), y la mecánica del juego (Figura 2) se 
http://doi.org/10.15359/ree.25-1.2

apoyó en la gran capacidad que tiene la niñez de aprender sobre las criaturas, artificiales o naturales, como puede evidenciarse por el hecho de que estudiantes de 8 años fueron capaces de identificar casi el 80\% de una muestra extraída de 150 especies "artificiales" de Pokémon (Balmford et al., 2002). Asimismo, la visualización de los animales durante el juego puede llevar a una identificación en la naturaleza, la cual es muy similar a la identificación a partir de fotografías (Palmberg et al., 2015).

Por su parte, el juego utiliza información ecológica y taxonómica para simular las posibles relaciones tróficas de diferentes grupos de animales (insectos, arácnidos, anfibios, reptiles, aves y mamíferos). Cada una de las 88 cartas contiene la fotografía de un animal (con la excepción de 8 comodines con fotografías de ecosistemas colombianos), el nombre científico, el nombre común en inglés y español, y un octágono con información del tamaño en centímetros y la dieta del animal. Todos los animales representados en las cartas son nativos de Colombia, con algunas especies endémicas.

Figura 2: Dinámica del juego

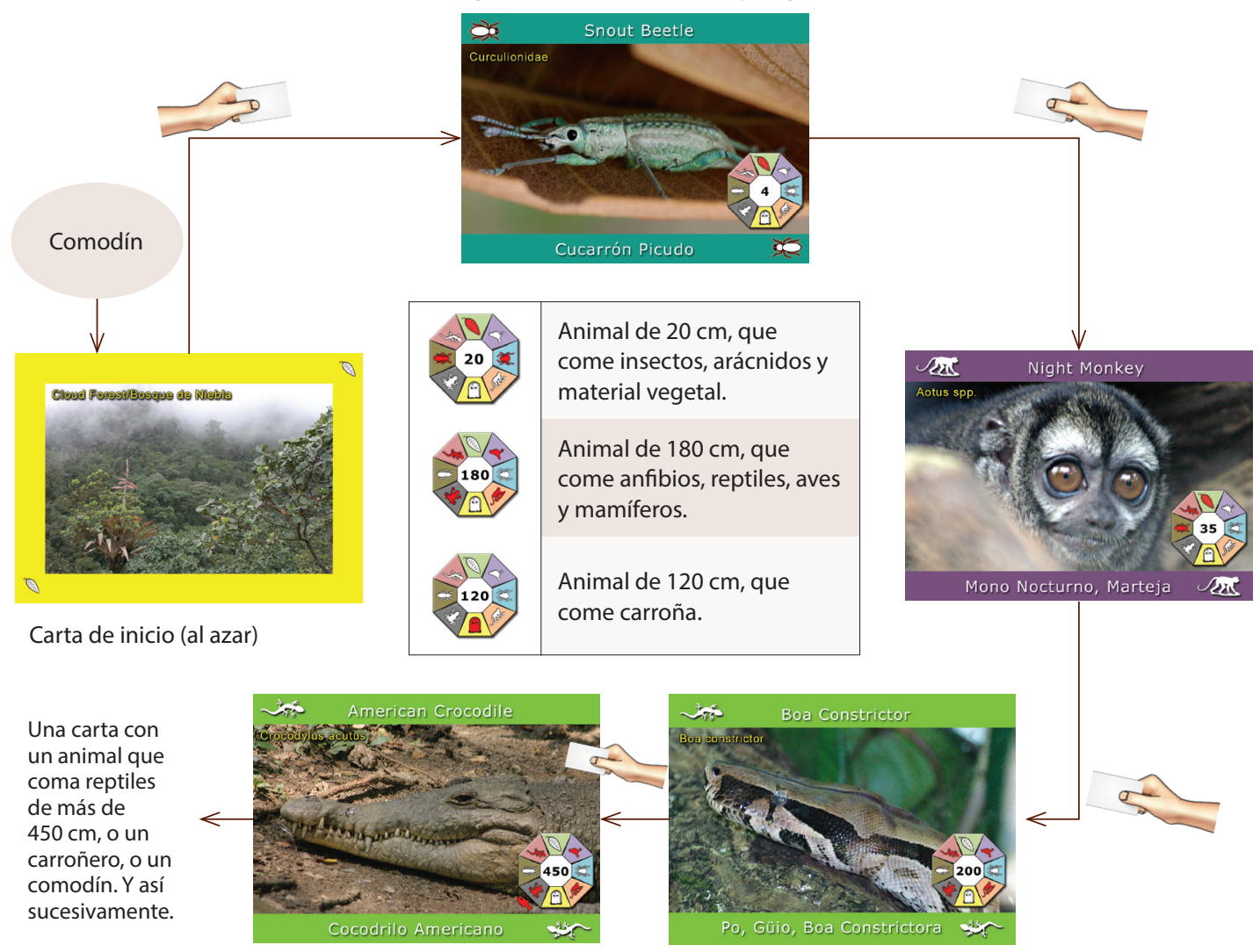

Nota: Elaboración propia. 
http://doi.org/10.15359/ree.25-1.2

http://www.una.ac.cr/educare

educare@una.ac.cr

\section{Resultados}

Los animales nativos mencionados por el estudiantado fueron asignados a una de las tres categorías taxonómicas etnobiológicas seleccionadas, mientras que los animales no nativos fueron excluidos del análisis. En comparación con las categorías específica y formas de vida, la categoría genérica tuvo el mayor número de menciones. En pre-juego versus post-juego hubo un aumento en el número de veces que fueron mencionados los taxones de las categorías genérica ( 2293 vs. 2505 ) y específica (400 vs. 706). Por su parte, hubo una disminución en el número de veces que se mencionaron las formas de vida (101 vs. 89) (Figura 3).

Figura 3: Número de veces que se mencionaron taxones pertenecientes a las formas de vida y a las categorías genérica y específica, pre-juego y post-juego

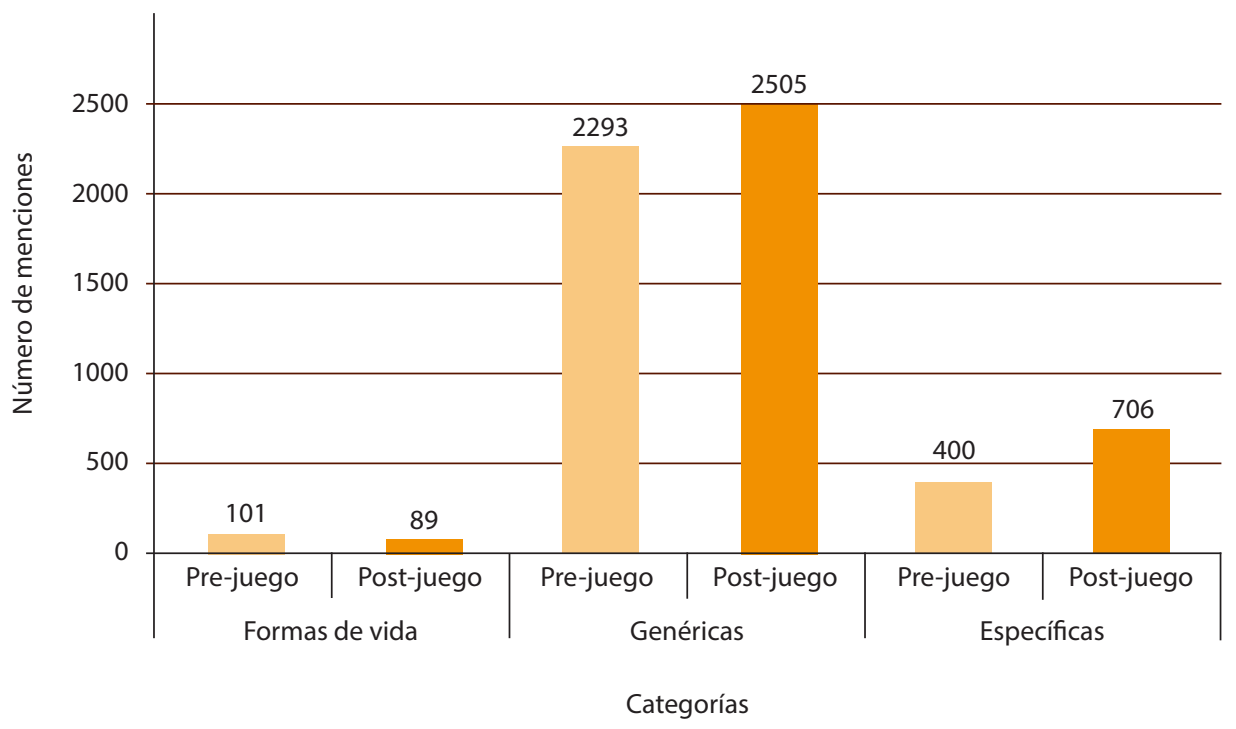

Nota: Elaboración propia.

En pre-juego y post-juego se mencionaron dos formas de vida (peces y serpientes). En la categoría genérica se mencionaron 82 taxones pre-juego y 104 post-juego, para un total de 115 taxones diferentes. Asimismo, en la categoría específica se mencionaron 24 taxones pre-juego y 53 post-juego, para un total de 64 taxones diferentes (Figura 4). 
http://doi.org/10.15359/ree.25-1.2

Figura 4: Número de diferentes taxones pertenecientes a las formas de vida y a las categorías genérica y específica, pre-juego y post-juego

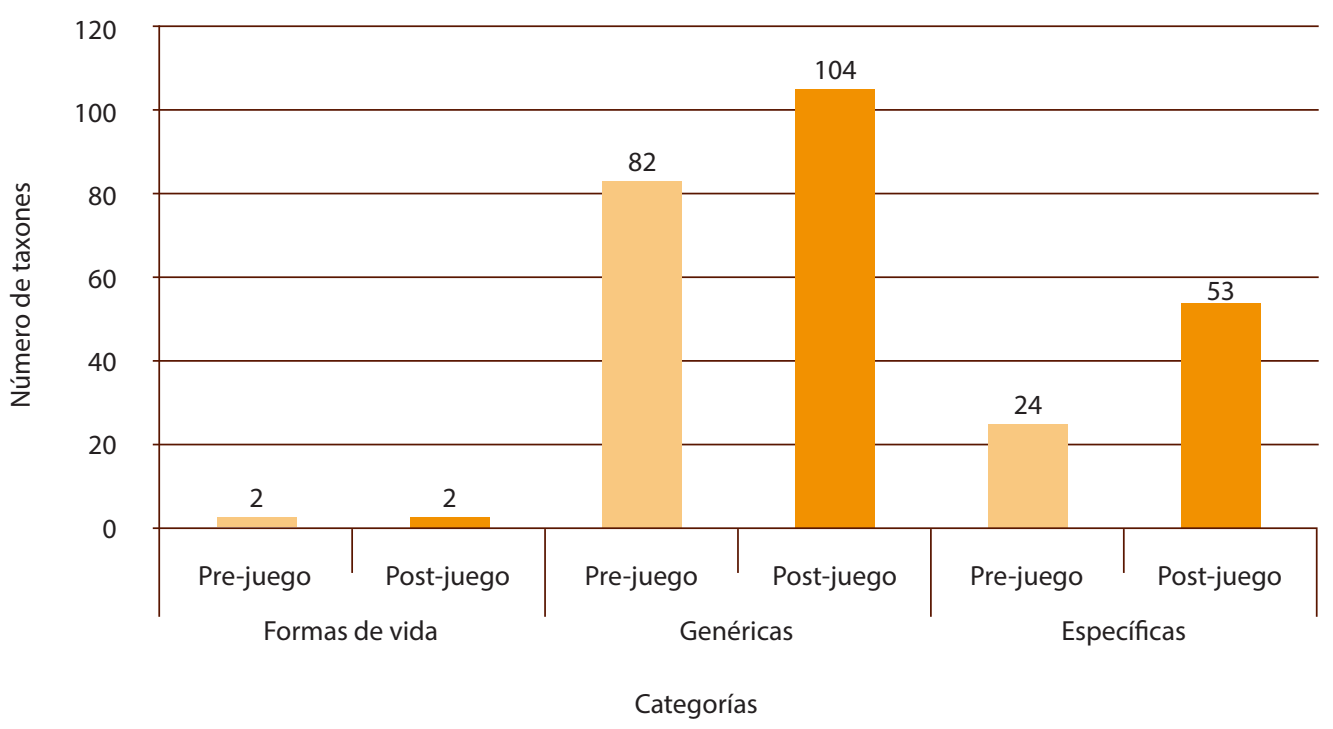

Nota: Elaboración propia.

\section{Discusión}

Es importante tener en cuenta que la niñez adquiere gradualmente las formas de vida "peces", "serpientes", "aves", "bichos" y "mamíferos", en su orden, entre los 3 y los 8 años de edad (Chase, 1979, citado en Brown, 1979), y a los 10 años de edad los niños y las niñas ya tienen un conocimiento elaborado de los animales (Johnson y Carey, 1998). Por consiguiente, puede esperarse que el estudiantado incluido en este estudio tenga al menos un conocimiento básico de la fauna nativa de Colombia.

Por otro lado, mientras el reconocimiento de los objetos cotidianos suele presentarse primero en el nivel básico y luego se expande a los niveles superordinados y subordinados, el grado de experticia cambia el punto de entrada del proceso al nivel subordinado (Belke et al., 2010), es decir, mientras el público general reconoce inicialmente un animal en una categoría genérica (por ejemplo, tití), una bióloga tendría un punto de entrada del proceso en una categoría específica (por ejemplo, tití pigmeo). En este sentido, aunque se observaron cambios favorables pre-juego vs. post-juego en el número de menciones (101 vs. 89 ) de las formas de vida y en el número de menciones y el número de taxones (2293 vs. 2505 y 82 vs. 104, respectivamente) de la categoría genérica, quizás el mayor impacto se dio en la categoría específica, en la que el número de menciones fue de casi el doble (400 vs. 706 ) y el número de taxones fue más del doble (24 vs. 53) después de jugar. Si se tiene en cuenta el punto de 
http://doi.org/10.15359/ree.25-1.2

http://www.una.ac.cr/educare

educare@una.ac.cr

entrada respecto al proceso de reconocimiento de los animales, este cambio en la categoría específica podría indicar un mayor grado de experticia en la identificación y clasificación de los animales, que ciertamente es un resultado anhelado para muchas intervenciones de educación ambiental sobre biodiversidad. Por otra parte, en este estudio, la categoría genérica fue la más abundante en número y tipo de taxones mencionados por los grupos participantes, lo cual también se presenta en la mayoría de taxonomías folk, las cuales suelen incluir cerca de 500 taxones (Hilgert, 2007).

En el presente estudio, el estudiantado incluyó un total de 115 taxones en la categoría genérica, que en comparación con los 500 taxones presentes en la categoría genérica de la mayoría de taxonomías folk parece escaso; sin embargo, no debe olvidarse que en general la niñez tiene un conocimiento escaso de los animales (Paraskevopoulos et al., 1998; LindemannMatthies y Bose, 2008), y los estudios etnobiológicos suelen entrevistar personas adultas o grupos de diferentes edades (Kane, 2015), quienes tendrían un mayor conocimiento de la flora y la fauna en comparación con la niñez. Además, aunque podría considerarse que 115 taxones en la categoría genérica son pocos, si se tiene en cuenta la participación de estudiantes de colegios rurales, ya que en comparación con la niñez de las ciudades, la de entornos rurales se muestra más interesada y conoce más de los animales (Kellert, 1984) y, en teoría, tiene un mayor reconocimiento de las especies por su nivel de dependencia de los recursos naturales y la frecuencia de su interacción con el ambiente (Campos et al., 2013), existe una creciente convergencia en los estilos de vida de poblaciones urbanas y rurales (Huddart-Kennedy et al., 2009), lo cual podría hacer que la niñez de estos dos tipos de entorno tengan conocimientos y actitudes similares frente a los animales.

Desde otro punto de vista, los resultados obtenidos en el presente estudio son alentadores, si se comparan con los obtenidos en un estudio suizo que encuestó a más de 4000 personas entre 8 y 16 años sobre los organismos en su entorno inmediato, en el cual fueron nombrados en promedio seis animales y cinco plantas, y taxones inespecíficos como "aves", "árboles" y "pastizales" estuvieron entre los más nombrados en todos los grupos etarios (LindemannMatthies, 2002). Este escaso conocimiento de la fauna no solo refleja la rápida disminución del contacto diario de la niñez con la naturaleza, sino también afecta la apreciación del público por el valor del mundo natural (Soga et al., 2016).

Si bien es cuestionable que la niñez de una urbe de cultura occidental como Bogotá y sus alrededores pueda tener una verdadera taxonomía folk, ya que la cultura y la experiencia (exposición a la naturaleza) juegan un papel importante en el desarrollo del pensamiento biológico folk (Ross et al., 2003), el presente estudio no se centró en la clasificación que la niñez hacía de los animales, sino que utilizó las categorías de la taxonomía folk para analizar los cambios cualitativos observados en la manera que las personas participantes nombraban los animales que conocían de la fauna nativa. 
http://doi.org/10.15359/ree.25-1.2

Cuando las personas desconocen el nombre de las plantas y los animales, es frecuente que los términos menos específicos, como las formas de vida (es decir, peces, aves, mamíferos, etc.), aumenten y se vuelvan cada vez más relevantes, y esta adición de las formas de vida a los lenguajes indica una disminución general del interés y de la preocupación respecto al mundo de los animales (Brown, 1982). Por consiguiente, una reducción en la mención de las formas de vida, al igual que un aumento en la mención y variedad de las categorías etnobiológicas "genérica" y "específica", deberían ser tanto el parámetro de evaluación como el objetivo de las estrategias de educación ambiental enfocadas en mejorar el conocimiento de la biodiversidad entre los niños, las niñas y el público general.

En cuanto al juego de cartas sobre biodiversidad, cabe mencionar que el estudiantado pedía los juegos para usarlos en el descanso, e incluso para llevarlos a casa. De hecho, el juego de cartas logró convertirse en una actividad popular durante el descanso de la escuela. Este alto grado de aceptación del juego permitió que niños y niñas se sumergieran en su mecánica, y esto, a su vez, hizo más probable que vieran las situaciones desde la perspectiva presentada en el juego, es decir que tuvieran una mayor probabilidad de adquirir la actitud estimulada en este (Ruggiero, 2015). En el contexto del juego, una rápida asociación de la fotografía con el nombre y las características de la especie aumentaba las probabilidades de ganar, una motivación que llevaría a mejorar las habilidades de identificación de especies en un entorno natural.

\section{Conclusiones y recomendaciones}

Dado que el conocimiento de los seres vivos entre la niñez y el público general se relaciona más con un saber popular que con uno científico, la taxonomía folk ofrece un mejor marco de referencia para diseñar, implementar y evaluar las estrategias de educación ambiental enfocadas en mejorar el conocimiento de la biodiversidad.

Ya que las estrategias de educación ambiental relacionadas con la biodiversidad deben responder a las necesidades y preferencias de la población objetivo, la implementación del juego durante el descanso de la escuela, además de brindar una alternativa de educación y diversión para la niñez, no interfiere con la ocupada agenda del personal docente en las instituciones. Esto deja ver que el juego puede ser una herramienta ideal para la educación ambiental en escenarios escolares. Por otra parte, la popularidad de este juego dentro de la población estudiantil durante el descanso de la escuela indicaría que la oferta de juegos educativos al estudiantado como alternativa a las actividades como el deporte y el ocio es una gran oportunidad que debe explorarse.

Para terminar, en el marco de la taxonomía folk, esta estrategia de educación ambiental basada en el juego logró lo que podría considerarse un resultado ideal para las intervenciones educativas relacionadas con el conocimiento de la biodiversidad, ya que obtuvo una disminución 
http://doi.org/10.15359/ree.25-1.2

http://www.una.ac.cr/educare

educare@una.ac.cr

en el número de menciones de formas de vida y un aumento en la composición y abundancia de los taxones en las categorías genérica y específica. Asimismo, los resultados de este estudio destacan la importancia de diseñar e implementar estrategias de educación ambiental que puedan generar una reconexión entre el conocimiento de la niñez y su contexto ecológico.

\section{Referencias}

Anderson, J. L. (2001). Stone-Age minds at work on 21 st century science: How cognitive psychology can inform conservation biology. Conservation in Practice, 2(3), 18-27. https:// doi.org/10.1111/j.1526-4629.2001.tb00013.x

Balmford, A., Clegg, L., Coulson, T. y Taylor, J. (2002). Why conservationists should heed Pokémon. Science, 295(5564), 2367. https://doi.org/10.1126/science.295.5564.2367b

Beaudreau, A. H., Levin, P. S. y Norman, K. C. (2011). Using folk taxonomies to understand stakeholder perceptions for species conservation. Conservation Letters, 4(6), 451-463. https://doi.org/10.1111/j.1755-263X.2011.00199.x

Belke, B., Leder, H., Harsanyi, G. y Carbon, C. C. (2010). When a Picasso is a "Picasso": The entry point in the identification of visual art. Acta Psychologica, 133(2), 191-202. https://doi. org/10.1016/j.actpsy.2009.11.007

Berlin, B., Breedlove, D. E. y Raven, P. H. (1973). General principles of classification and nomenclature in folk biology. American Anthropologist, 75(1), 214-242. https://doi. org/10.1525/aa.1973.75.1.02a00140

Bevilacqua, M., Ciarapica, F. E., Mazzuto, G. y Paciarotti, C. (2015). "Cook \& Teach": Learning by playing. Journal of Cleaner Production, 106, 259-271. http://doi.org/10.1016/j. jclepro.2014.11.085

Brown, C. H. (1979). Folk zoological life-forms: Their universality and growth. American Anthropologist, 81(4), 791-817. https://doi.org/10.1525/aa.1979.81.4.02a00030

Brown, C. H. (1982). Folk zoological life-forms and liguistic marking. Journal of Ethnobiology, 2(1), 95-112. https://ethnobiology.org/journal/\%5Bfield volume-raw\%5D-57

Cabral de Oliveira, J. (2008). Classificações em cena. Algumas formas de classificações das plantas cultivadas pelos Wajãpi do Amapari (AP). En M. Lenaerts y A. M. Spadafora (Eds.), Pueblos indígenas, plantas y mercados. Amazonía y Gran Chaco (pp. 29-58). Zeta Books. https:// www.academia.edu/31697104/Pueblos ind\%C3\%ADgenas plantas y mercado Amazonia_e_Gran_Chaco 
http://doi.org/10.15359/ree.25-1.2

Campos, C. M., Nates, J. y Lindemann-Matthies, P. (2013). Percepción y conocimiento de la biodiversidad por estudiantes urbanos y rurales de las tierras áridas del centro-oeste de Argentina. Ecologia Austral, 23(3), 174-183. www.scielo.org.ar/pdf/ecoaus/v23n3/ v23n3a06.pdf

Ferreira, E. N., da S Mourão, J., Rocha, P. D., Nascimento, D. M. y da S Q Bezerra, D. M. M. (2009). Folk classification of the crabs and swimming crabs (Crustacea - Brachyura) of the Mamanguape river estuary, Northeastern - Brazil. Journal of Ethnobiology and Ethnomedicine, 5(22), 1-11. https://doi.org/10.1186/1746-4269-5-22

Gómez, M. F. y Moreno, L. A. (2016). Biodiversidad 2015 en cifras. En M. F. Gómez, L. A. Moreno, G. I. Andrade y C. Rueda (Eds.), Biodiversidad 2015. Estado y tendencias de la biodiversidad continental de Colombia (pp. 10-13). Instituto Alexander von Humboldt. http://repository. humboldt.org.co/bitstream/handle/20.500.11761/9305/IAVH_Biodiversidad_2015_WEB. pdf? sequence $=1$ \&isAllowed $=y$

Goyenechea, I. (2007). Sistemática: Su historia, sus métodos y sus aplicaciones en las serpientes del género Conopsis. Ciencia ergo sum, 14(1), 54-62. https://www.redalyc.org/articulo. oa?id=10414107

Hilgert, N. (2007). La etnobotánica como herramienta para el estudio de los sistemas de clasificación tradicionales. En A. Contreras-Ramos, C. Cuevas-Cardona, I. Goyenechea y U. Iturbe (Eds.), La sistemática, base del conocimiento de la biodiversidad (pp. 103-111). Universidad Autónoma del Estado de Hidalgo. https://www.researchgate.net/profile/ Irene Goyenechea2/publication/268817766 La sistematica base del conocimiento de la biodiversidad/links/5478b9010cf293e2da2b2b3c/La-sistematica-base-delconocimiento-de-la-biodiversidad.pdf

Huddart-Kennedy, E., Beckley, T. M., McFarlane, B. L. y Nadeau, S. (2009). Rural-Urban differences in environmental concern in Canada. Rural Sociology, 74(3), 309-329. https://doi. org/10.1526/003601109789037268

Johnson, S. C. y Carey, S. (1998). Knowledge enrichment and conceptual change in folkbiology: Evidence from Williams syndrome. Cognitive Psychology, 37(2), 156-200. https://doi. org/10.1006/cogp.1998.0695

Kane, S. C. (2015). Bird names and folklore from the Emberá (Chocó) in Darién, Panamá. Ethnobiology Letters, 6(1), 32-62. https://doi.org/10.14237/ebl.6.1.2015.226

Kellert, S. R. (1984). Attitudes toward animals: Age-Related development among children. En M. W. Fox y L. D. Mickley (Eds.), Advances in animal welfare science (Vol. 85, pp. 43-60). The Humane Society of the United States. 
http://doi.org/10.15359/ree.25-1.2

http://www.una.ac.cr/educare

educare@una.ac.cr

Lindemann-Matthies, P. (2002). The Influence of an educational program on children's perception of biodiversity. The Journal of Environmental Education, 33(2), 22-31. https:// doi.org/10.1080/00958960209600805

Lindemann-Matthies, P. y Bose, E. (2008). How many species are there? Public understanding and awareness of biodiversity in Switzerland. Human Ecology, 36(5), 731-742. https://doi. org/10.1007/s10745-008-9194-1

Mace, G. M. (2004). The role of taxonomy in species conservation. Philosophical Transactions of the Royal Society B, 359(1444), 711-719. https://doi.org/10.1098/rstb.2003.1454

Palmberg, I., Berg, I., Jeronen, E., Kärkkäinen, S., Norrgård-Sillanpää, P., Persson, C., Vilkonis, R. y Yli-Panula, E. (2015). Nordic-Baltic student teachers' identification of and interest in plant and animal species: The importance of species identification and biodiversity for sustainable development. Journal of Science Teacher Education, 26(6), 549-571. https://doi. org/10.1007/s10972-015-9438-z

Paraskevopoulos, S., Padeliadu, S. y Zafiropoulos, K. (1998). Environmental knowledge of elementary school students in Greece. The Journal of Environmental Education, 29(3), 5560. https://doi.org/10.1080/00958969809599119

Petrovska, S., Sivevska, D. y Cackov, O. (2013). Role of the game in the development of preschool child. Procedia - Social and Behavioral Sciences, 92, 880-884. http://doi.org/10.1016/j. sbspro.2013.08.770

Raven, P. H., Berlin, B. y Breedlove, D. E. (1971). The Origins of taxonomy. Science, 174(4015), 1210-1213. https://doi.org/10.1126/science.174.4015.1210

Rosch, E., Mervis, C. B., Gray, W. D., Johnson, D. M. y Boyes-Braem, P. (1976). Basic objects in natural categories. Cognitive Psychology, 8(3), 382-439. https://doi.org/10.1016/0010$\underline{0285(76) 90013-X}$

Ross, N., Medin, D., Coley, J. D. y Atran, S. (2003). Cultural and experiential differences in the development of folkbiological induction. Cognitive Development, 18(1), 25-47. https://doi. org/10.1016/S0885-2014(02)00142-9

Rozzi, R., Massardo, F., Silander, J. Jr., Anderson, C. y Marín, A. (2003). Capítulo Il. Conservación biocultural y ética ambiental en el extremo austral de América: Oportunidades y dificultades para el bienestar ecosocial. En E. Figueroa y J. A. Simonetti (Eds.), Globalización y biodiversidad: Oportunidades y desafíos para la sociedad chilena (pp. 51-85). Editorial Universitaria. 
http://doi.org/10.15359/ree.25-1.2 http://www.una.ac.cr/educare educare@una.ac.cr

Ruggiero, D. (2015). The effect of a persuasive social impact game on affective learning and attitude. Computers in Human Behavior, 45, 213-221. http://doi.org/10.1016/j. chb.2014.11.062

Soga, M., Gaston, K. J., Koyanagi, T. F., Kurisu, K.y Hanaki, K. (2016). Urban residents'perceptions of neighbourhood nature: Does the extinction of experience matter? Biological Conservation, 203, 143-150. https://doi.org/10.1016/j.biocon.2016.09.020

Taylor, J. L. (1983). Guía sobre simulación y juegos para la educación ambiental. ORELAC. https:// es.calameo.com/read/00090832793ca99569904

Tun Tun, C. E. (2016). Taxonomía popular y taxonomía científica: ¿Hay correspondencia? Desde El Herbario: CICY, 8, 174-176. http://www.cicy.mx/Documentos/CICY/Desde Herbario/2016/2016-11-10-TunTun-Taxonomia-popular-y-taxonomia-cientifica.pdf

Turkay, S., Adinolf, S. y Tirthali, D. (2012). Collectible card games as learning tools. Procedia-Social and Behavioral Sciences, 46, 3701-3705. https://doi.org/10.1016/j.sbspro.2012.06.130

van Eck, R. (2006). Digital game-based learning: It's not just the digital natives who are restless. Educause Review, 41(2), 16-30. http://doi.org/10.1145/950566.950596

Zubin, D. y Köpcke, K. M. (1986). Gender and folk taxonomy: The indexical relation between grammatical and lexical categorization. En C. G. Craig (Ed.), Noun classes and categorization: Proceedings of a Symposium on Categorization and Noun Classification (pp. 139-180). John Benjamins. https://doi.org/10.1075/tsl.7.12zub 